\title{
TROIS PROBLĖMES NON LINÉAIRES DE L'ÉTUDE MÉCANIQUE DE LA RUPTURE DES MATÉRIAUX
}

\author{
H. B. BUI \\ Laboratoire de Mécanique des Solides et Centre des \\ Renardières, Electricité de France, 77250 Moret-sur-Loing, France \\ et

\section{K. DANG VAN} \\ Laboratoire de Mécanique des Solides, \\ Ecole Polytechnique et Ecole Nationale Supérieure des Mines de Paris, \\ 75005 Paris, France
}

\begin{abstract}
Résumé. - Trois problèmes de mécanique non linéaire de la rupture sont examinés.
La propagation de la fissure est étudiée dans le cas d'un critère de rupture s'exprimant à l'aide d'une relation entre ouverture de fissure et force de cohésion. Deux équations intégrales non linéaires sont obtenues.

Partant d'une loi de comportement du type $\varepsilon(\sigma)$, on propose la nouvelle intégrale $I$ indépendante du contour. Cette intégrale formulée récemment par Bui est complémentaire de l'intégrale J de Rice.

L'analyse numérique tridimensionnelle de quelques exemples de structures fissurées, ayant pour comportement la loi élasto-plastique incrémentale, permet d'étudier le développement des zones plastiques. Les facteurs d'intensité des contraintes $K_{I}$ sont discutés.
\end{abstract}

\begin{abstract}
This paper is concerned with three non-linear problems of fracture mechanics.
The first is related to the crack propagation for which non-linear integral equations are obtained for models of crack with cohesive forces.

The second is related to the new path independent integral $I$, which is associated with the stressstraim law $\varepsilon(\sigma)$. This integral given recently by Bui is dual to the path independent integral $\mathfrak{J}$ given by Rice.

The third is related to the numerical three-dimensional analysis of cracked bodies ; plastic flow law is adopted for investigating the development of plastic zones. Stress intensity factors $K_{I}$ are discussed in connexion with the three-dimensional analysis.
\end{abstract}

1. Introduction. - L'étude de la rupture est bien développée pour les matériaux fragiles, en particulier les métaux à très haute résistance. L'examen des conditions de validité de la théorie montre que l'application à des structures moins fragiles est possible pourvu que d'une part la déformation plastique reste faible et confinée en fond de fissure, et que d'autre part l'état de déformation soit sensiblement plan.

Un des problèmes examinés ici est de vérifier les deux conditions précédentes par un calcul élastoplastique tridimensionnel. Examiner ce problème, c'est aborder un des aspects non linéaires de l'étude mécanique de la rupture. L'étude de la propagation brutale montre que le critère de rupture de Griffith introduit lui-même la non-linéarité dans la réponse de la structure au chargement. Un autre exemple fondé sur le modèle des forces de cohésion confirmera ce point de vue.
Mais la cause principale de non-linéarité provient du comportement mécanique. Deux types de loi sont envisageables :

- la loi du type $\varepsilon(\sigma)$ sans être forcément linéaire, analogue à celle $\sigma(\varepsilon)$ utilisée par Rice [1-2]

— la loi élasto-plastique incrémentale.

L'étude de la première loi conduit à la formulation d'une nouvelle intégrale $I$ indépendante du contour d'intégration. Cette intégrale formulée récemment par Bui [3] est complémentaire de l'intégrale $\mathfrak{J}$ de Rice. Les deux approches conduisent aux méthodes numériques d'approximation de l'énergie potentielle.

L'étude de la seconde loi a été faite numériquement pour des structures simples, plaque en tension, éprouvettes en flexion pure et en flexion à trois appuis. Cette étude faite dans le cas tridimensionnel a deux objectifs : le premier est de connaître les zones plas- 
tiques et le second de savoir dans quelle mesure l'état plan est respecté pour pouvoir définir un facteur d'intensité $K_{I}$. L'étude tridimensionnelle fait apparaître plusieurs manières de définir ce dernier facteur.

2. Caractère non linéaire de la propagation de la fissure. - La rupture fragile peut être interprétée de façon satisfaisante par le modèle de Griffith [4]. Le critère local de Griffith postule que l'extension d'une fissure existante de surface $S$, par une nouvelle surface $\mathrm{d} S(\mathrm{~d} S=2 \mathrm{~d} l$ par unité d'épaisseur, $l$ longueur de fissure) exige une énergie de surface $\gamma \mathrm{d} S$. Lorsque la rupture a lieu sous la charge constante $F_{\mathrm{c}}$, la valeur de celle-ci est déterminée par un bilan énergétique du type :

$$
F_{\mathrm{c}} \mathrm{d} u=\mathrm{d} W_{\text {elast }}+\gamma \mathrm{d} S .
$$

L'élasticité étant linéaire, l'énergie élastique est égale à la moitié du travail des forces extérieures $F_{\mathrm{c}} \mathrm{d} u=2 \mathrm{~d} W_{\text {élast. }}$ Il en résulte le critère global de Griffith :

$$
\frac{\mathrm{d} W_{\text {elast }}}{\mathrm{d} l}=2 \gamma
$$

La force critique $F_{\mathrm{c}}$ est alors simplement déterminée par cette dernière relation.

Or, si on regarde toute la réponse de la structure au chargement $F$, la propagation de la fissure comprise, on constate que cette réponse n'est pas linéaire. La courbe $O A B$ de la figure 1 a en effet un point anguleux. La réponse n'est linéaire qu' " avant » la propagation.

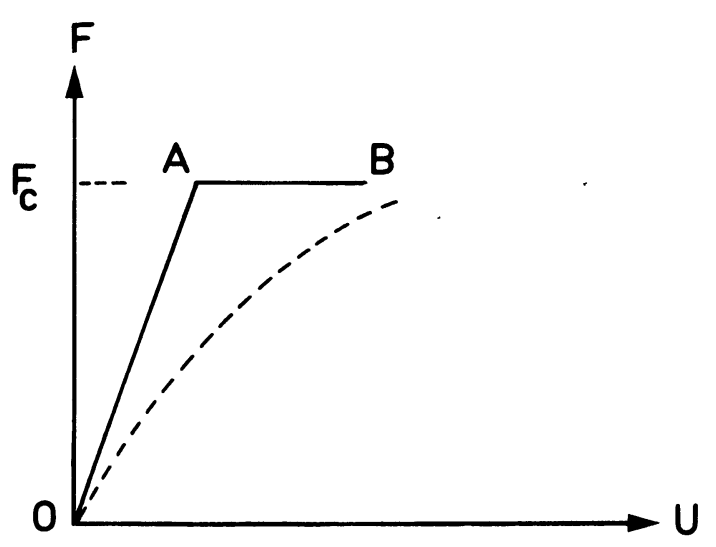

FIG. 1.

Cette situation n'est pas sans rappeler le problème bien connu du flambage des poutres.

Examinons maintenant un autre critère de rupture sur l'exemple suivant : soit une grande plaque épaisse affaiblie par une petite fissure centrale, soumise à la tension $F$ perpendiculairement à la fissure, en déformation élastique plane. Travaillant en mode I, nous pouvons supposer que l'ouverture de fissure $u$ et la contrainte normale $T$ dans le plan de la fissure sont reliées par des relations du type $u(T)$ ou $T(u)$. De telles relations utilisées par plusieurs auteurs [5-6] sont associées aux forces de cohésion généralisées. Après une phase croissante, la courbe $T(u)$ par exemple décroît par suite de la décohésion du matériau. Physiquement, la décroissance de $T$ est due, soit à la rupture de liaison atomique dans le cas fragile, soit à la dégradation du métal par formation de microfissures et de cavités dans le cas ductile. Dans les deux cas, la courbe $T(u)$ est typiquement non linéaire.

Avec ce critère de rupture, il n'est pas possible d'écrire un bilan énergétique analogue à (1). On doit calculer directement la réponse de la structure sous la forme d'une relation entre la traction $F$ et l'ouverture $u_{0}$ au centre. Le principe du calcul repose sur la relation entre l'ouverture $u(x)$ de la fissure occupant l'axe $O x$, la tension normale $T(x)$ sur l'axe $O x$ et la traction à l'infini $F$, ou bien la relation entre $u(x)$ et la tension $\sigma(x)=T-F$ d'un problème équivalant avec la traction nulle à l'infini. Cette dernière relation peut être fournie par la théorie de l'élasticité plane. Elle est donnée explicitement dans la référence [7], sous forme d'équations intégrales $u^{\prime}=\mathfrak{L}(\sigma)$ ou $\sigma=\mathfrak{L}^{-1}\left[u^{\prime}\right]$. Explicitant ces dernières et remplaçant $\sigma$ par $T-F$, nous obtenons les deux équations intégrales réciproques l'une de l'autre (cf. (7)) :

$$
\begin{gathered}
-F+T[u(x)]=\frac{-E}{2 \pi\left(1-v^{2}\right)} \int_{-\infty}^{+\infty} \frac{\mathrm{d} u}{\mathrm{~d} t} \frac{\mathrm{d} t}{t-x} \\
\frac{\mathrm{d}}{\mathrm{d} x}[u(T)]=\frac{2\left(1-v^{2}\right)}{\pi E} \int_{-\infty}^{+\infty}[-F+T(t)] \frac{\mathrm{d} t}{t-x} .
\end{gathered}
$$

L'une ou l'autre de ces équations permet de trouver la répartition $u(x)$ à partir de la donnée $F$, donc finalement la relation souhaitée entre $F$ et $u_{0}=u(0)$. Soumise à la traction $F$, la structure donne une réponse non linéaire parce que les équations (2) et (3) le sont justement à cause de $u(T)$ et de $T(u)$. C'est ce que représente schématiquement la courbe en pointillé de la figure 1. Aucun bilan énergétique n'est en mesure de fournir la force critique $F_{\mathrm{c}}$. Celle-ci ne peut être obtenue que par la résolution pas à pas d'une équation intégrale non linéaire.

Pour le même problème, Anderson et Bergkvist [6] ont proposé l'équation suivante :

$u[T(x)]=-\frac{2\left(1-v^{2}\right)}{\pi E} \int_{-\infty}^{+\infty}[-F+T(t)] \log |t-x| \mathrm{d} t$,

c'est une forme légèrement différente de (3), c'est-à-dire, déjà intégrée une fois en $x$. Leur analyse numérique montre que l'ouverture de fissure et la longueur de fissure, c'est-à-dire l'intervalle dans lequel la contrainte est nulle, croissent avec la charge, si bien que la notion même d'une progagation brutale connue en théorie fragile disparaît. La propagation est donc continue et progressive.

Cet exemple illustre peut-être ce qui se passe dans la rupture des métaux, bien que le problème soit un peu différent parce qu'il faut considérer le comportement 
plastique et non élastique, mais l'exemple est plus significatif avec le comportement élastique, justement parce qu'il montre que la non-linéarité est bien le fait du critère de rupture.

3. Intégrales indépendantes du contour $I$ et $\mathfrak{J}$. L'utilisation faite par Rice d'une loi de comportement du type $\sigma(\varepsilon)$ permet de simplifier beaucoup l'analyse mécanique des problèmes de fissures. Naturellement, une telle loi ne peut pas décrire la différence de comportement, à la charge et à la décharge. Aussi doit-on se limiter à des chargements monotones croissants et étudier plutôt le problème d'initiation de fissure que celui de la propagation qui entraîne des zones de décharge élastique en arrière $\mathrm{du}$ front de fissure.

Bui [3] a envisagé une loi de comportement du même type mais écrite sous la forme inversée $\varepsilon(\sigma)$. En étudiant une structure bidimensionnelle, affaiblie par une fissure de longueur $l$, soumise à des chargements comme indiqués sur la figure 2 , il montre qu'il existe une intégrale indépendante du contour d'intégration $\Gamma$ définie comme suit :

$$
I=\int_{\Gamma}\left[-U(\sigma) \mathrm{d} y+u_{\mathrm{i}} n_{\mathrm{j}} \frac{\partial \sigma_{\mathrm{ij}}}{\partial x} \mathrm{~d} s\right]
$$

avec

$$
U(\sigma)=\int_{0}^{\sigma} \varepsilon_{\mathrm{ij}}(\sigma) \mathrm{d} \sigma_{\mathrm{ij}}
$$

Cette intégrale possède la propriété :

$$
\begin{gathered}
I=-\frac{\mathrm{d} Q}{\mathrm{~d} l} \\
Q=-\int_{V} U \mathrm{~d} v+\int_{S U} u_{\mathrm{i}}^{\mathrm{d}} \sigma_{\mathrm{ij}} n_{\mathrm{j}} \mathrm{d} S .
\end{gathered}
$$

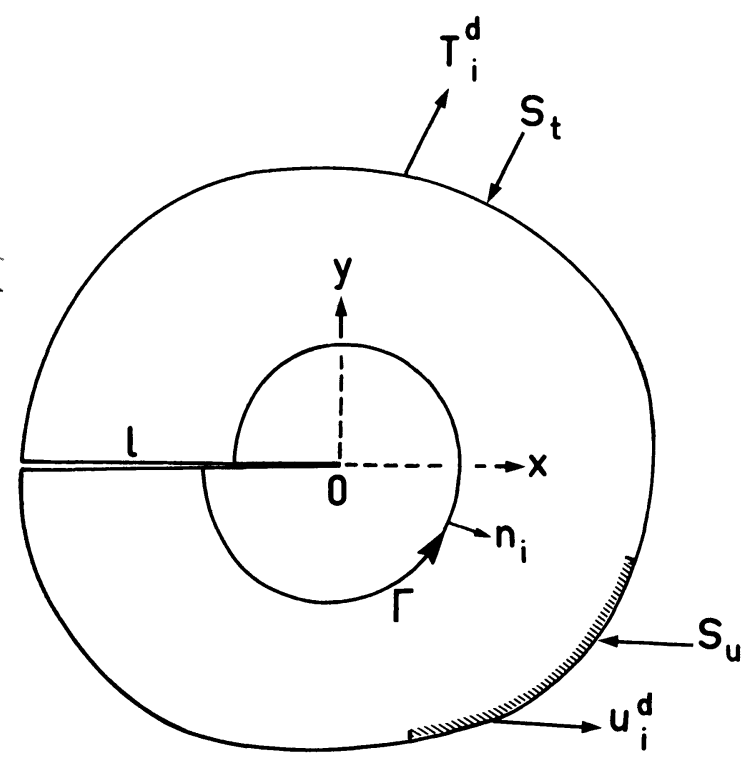

FIG. 2.
En d'autres termes, l'intégrale $I$ est le taux de décroissance de l'énergie potentielle complémentaire $Q(\sigma)$ du champ de contrainte, par rapport à l'accroissement de la longueur de fissure.

On voit immédiatement l'analogie avec les propriétés de l'intégrale $J$ de Rice, également indépendante du contour d'intégration $\Gamma$, en comparant les relations (5), (6), (7) et (8) aux suivantes :

$$
\begin{aligned}
& J \quad=\int_{\Gamma}\left[W(\varepsilon) \mathrm{d} y-\sigma_{\mathrm{ij}} n_{\mathrm{j}} \frac{\partial u_{\mathrm{i}}}{\partial x} \mathrm{~d} s_{\mathrm{i}}\right] \\
& W(\varepsilon)=\int_{0}^{\varepsilon} \sigma_{\mathrm{ij}}(\varepsilon) \mathrm{d} \varepsilon_{\mathrm{ij}} \\
& J \quad=-\frac{\mathrm{d} P}{\mathrm{~d} l} \\
& P \quad=\int_{V} W \mathrm{~d} v-\int_{S_{T}} I_{\mathrm{i}}^{\mathrm{d}} u_{\mathrm{i}} \mathrm{d} S .
\end{aligned}
$$

L'intégrale $\mathfrak{J}$ est le taux de décroissance de l'énergie potentielle $P(\varepsilon)$ du champ de déformation.

Il existe entre les deux formulations un rapport étroit. C'est que, grâce à l'identification du comportement, on peut écrire $U(\sigma)+W(\varepsilon)=\sigma_{\mathrm{ij}} \varepsilon_{\mathrm{ij}}$. D'où on peut déduire que $P(\varepsilon)=Q(\sigma)$ et $J=I$. Ainsi on aboutit à la même valeur du taux de décroissance de l'énergie par deux intégrales d'expression mathématique différentes.

Il faut toutefois souligner que l'identité $\mathfrak{J}=I$ n'est vraie que si les deux champs $\varepsilon$ et $\sigma$ sont solutions exactes du même problème mécanique. S'il n'en est pas ainsi, les valeurs approchées des potentiels $P$ et $Q$ correspondants sont différentes, et les intégrales $J$ et $I$ également. En outre, les intégrales approchées dépendent du contour d'intégration.

Plus précisément, considérons deux types de solutions approchées, les unes de type cinématique $\varepsilon^{*}$, les autres de type statique $\sigma^{* *}$. Les premières peuvent être obtenues par la méthode des éléments finis. Les secondes sont surtout utilisées dans la théorie des charges limites (8). Les potentiels $P^{*}$ obtenus de (12) et $Q^{* *}$ obtenus de (8) n'ont aucune raison d'être égaux puisque les solutions approchées $\varepsilon^{*}$ et $\sigma^{* *}$ sont construites indépendamment. D'ailleurs moyennant certaines hypothèses dites de convexité $(*)$, les potentiels $P^{*}$ et $Q^{* *}$, changés de signe, satisfont aux inégalités suivantes :

$$
-P^{*} \leqslant-P=-Q \leqslant-Q^{* *} .
$$

Ainsi la solution exacte - $P($ ou $-Q)$ se trouve majorée par $-Q^{* *}$ et minorée par $-P^{*}$. En fonction de la longueur de fissure $l$, la courbe exacte $-P(l)$ se trouve encadrée par les courbes appro-

(*) L'hypothèse de convexité de $W(\varepsilon)$ est :

$$
\mathrm{W}\left(\varepsilon^{*}\right)-W(\varepsilon) \geqslant \frac{\partial W(\varepsilon)}{\partial \varepsilon_{i \mathrm{j}}}\left[\varepsilon_{\mathrm{ij}}^{*}-\varepsilon_{\mathrm{ij}}\right] .
$$


chées fournies d'une part par l'approche de Rice et d'autre part par l'approche de Bui.

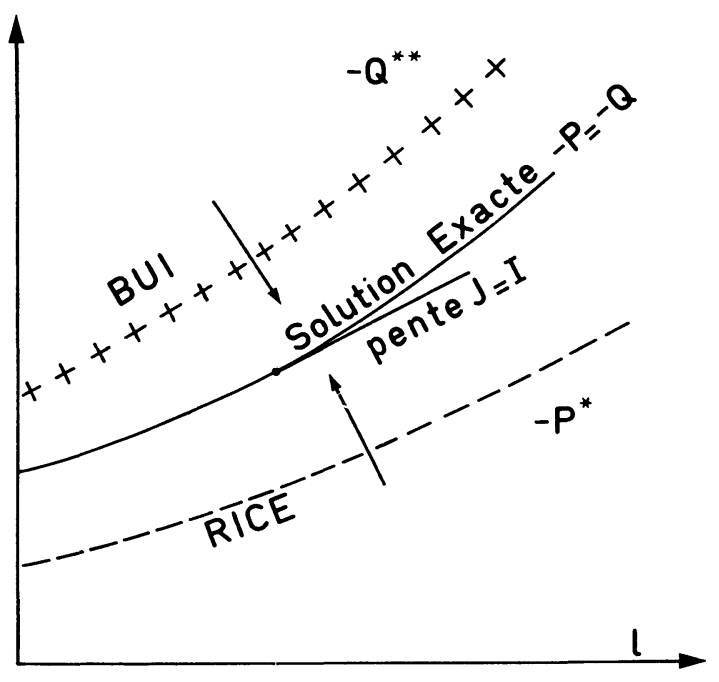

FIG. 3.

Les solutions numériques usuelles sont généralement du type cinématique et sont toutes situées en dessous de la courbe exacte $-P(l)$. Les meilleures solutions parmi elles sont évidemment celles qui donnent à $-P^{*}$ la plus grande valeur. Mais la connaissance d'une solution d'un type déterminé ne permet pas d'apprécier avec certitude la solution exacte. Pour y arriver, il est nécessaire de construire les solutions des deux types.

Les deux points de vue conduisant à $J$ et $I$ sont deux approches différentes mais complémentaires du même problème mécanique. Il s'agit en effet de l'étude faite sur la même géométrie, avec les conditions aux limites identiques, sur le même matériau dont la loi de comportement est simplement exprimée de façon différente. On a enfin le même critère local de rupture $\mathfrak{J}_{\mathrm{c}}=I_{\mathrm{c}}$, obtenu avec des contours petits en fond de fissure, car les deux intégrales sont identiques pour la solution exacte.

4. Analyse élasto-plastique tridimensionnelle. L'étude élasto-plastique des structures, même dépourvues de fissures, est très difficile. Les difficultés proviennent de ce que les lois de comportement sont incrémentales. En outre, les dernières font intervenir de façon complexe un certain nombre de paramètres d'écrouissage. C'est dire que la description correcte du comportement d'un métal donné soulève déjà en elle-même des problèmes.

Cependant, dans certaines circonstances, par exemple lorsque le chargement est monotone croissant, ou lorsqu'il n'y a pas de décharge à l'intérieur de la structure, des approximations raisonnables peuvent être utilisées : loi d'écrouissage isotrope, théorie de la plasticité de Hencky, etc...

Nous avons pris la loi incrémentale de PrandtlReuss [8] avec écrouissage isotrope pour étudier quelques structures simples : plaque à fissure centrale soumise à la traction [9], éprouvette à fissure latérale en flexion pure [10] et en flexion à trois appuis.
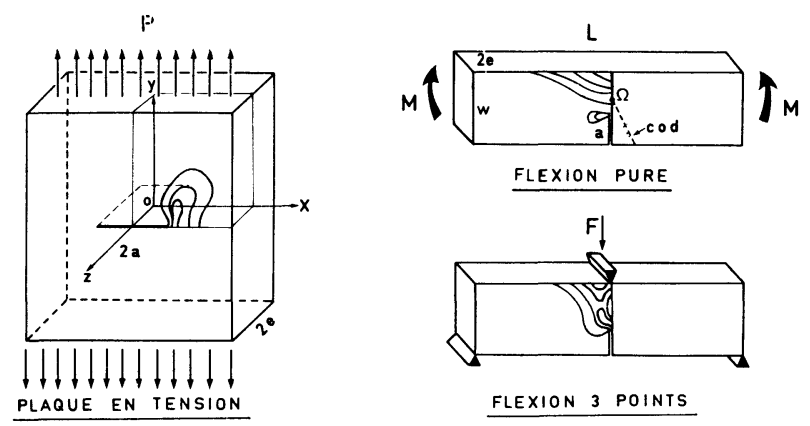

FIG. 4.

4.1 EQuations DE BASE. - Le système des équations à résoudre n'est pas linéaire à cause d'une part du critère de plasticité et d'autre part du processus incrémental. Avec les notations habituelles en plasticité, voici les équations :

$$
\text { (A) }\left\{\begin{array}{l}
\dot{\sigma}_{\mathrm{ij}, \mathrm{j}}=0 \\
\dot{\sigma}_{\mathrm{ij}}=\lambda \dot{\varepsilon}_{\mathrm{kk}} \dot{\delta}_{\mathrm{ij}}+2 \mu \dot{\varepsilon}_{\mathrm{ij}}-2 \mu \dot{\varepsilon}_{\mathrm{ij}}^{\mathrm{p}} \\
\dot{\varepsilon}_{\mathrm{ij}}=\frac{1}{2}\left(\dot{u}_{\mathrm{i}, \mathrm{j}}+\dot{u}_{\mathrm{j}, \mathrm{i}}\right) \\
\dot{\varepsilon}_{\mathrm{ij}}^{\mathrm{p}}=g(\sigma) s_{\mathrm{ij}} s_{\mathrm{hk}} \dot{\sigma}_{\mathrm{hk}} \quad(s: \text { déviateur de } \sigma)
\end{array}\right.
$$

avec les conditions suivantes :

(B) $\left\{\begin{array}{l}s_{\mathrm{ij}} \dot{\varepsilon}_{\mathrm{ji}}^{\mathrm{p}}>0, \text { si } f \equiv \frac{1}{2} s_{\mathrm{ij}} s_{\mathrm{ij}}=k^{2} \text { et } \dot{f}>0 \\ s_{\mathrm{ij}} \dot{\varepsilon}_{\mathrm{ji}}^{\mathrm{p}}=0, \text { si } \dot{f}<0 \text { ou si } f<k_{0}^{2}=\frac{\sigma_{0}^{2}}{3}\end{array}\right.$

$\sigma_{0}:$ limite élastique en traction.

L'inverse de $g(\sigma)$ est appelé module d'écrouissage $H(f)=1 / g$. C'est une fonction de $f$ que l'on détermine graphiquement par un essai de traction (suivant $\left.O x_{1}\right)$ :

$$
H(f)=\frac{4}{3} f \frac{\mathrm{d} \sigma_{11}}{\mathrm{~d} \varepsilon_{11}^{\mathrm{p}}} .
$$

Le matériau étudié est un acier inoxydable, dont la courbe de traction, déduite de la partie élastique, peut être approchée par la relation

$$
\sigma=\sigma_{0}+C\left(\varepsilon^{\mathrm{p}}\right)^{1 / n},\left(\sigma \equiv \sigma_{11}\right)
$$

avec $n$ un coefficient voisin de 2. Cette relation n'est valable que pour les petites déformations.

4.2 Méthode De CALCUL NUMÉRIQUe tRIDIMENSIONNEL. - Pour la plaque en traction et l'éprouvette en flexion pure, nous utilisons la méthode des différences finies combinée avec la technique de grilles successives de Ayres [11]. Pour l'éprouvette en flexion à trois appuis, nous utilisons la méthode des éléments finis classique avec toutefois une légère modification 
pour réaliser le calcul tridimensionnel. Le plan moyen $O x y$, en déformation plane, est divisé en éléments triangulaires, avec les déplacements $u$ et $v$ fonctions linéaires de $x$ et $y$ dans chaque triangle. Le déplacement tranversal $w$ dans le sens de l'épaisseur est un élément fini unique de la forme

$$
w(x, y, z)=G(x, y) \text { Signe }(z)\left(\frac{z}{e}\right)^{\alpha},
$$

où $\alpha$ est un scalaire positif convenablement choisi et $e$ la demi-épaisseur de l'éprouvette. Finalement en chaque nœud du plan $O x y$, il y a 3 inconnues $u, v, G$.

4.3 Discussions Des RÉSUltats. - Les zones plastiques en fond de fissure varient dans l'épaisseur de la plaque en tension, comme l'indique la figure 5 . Ces zones sont indiquées pour quatre incréments de charge de $15 \%$ chaque fois à partir d'une charge de référence $P_{0}$, avec laquelle débute la plastification dans la maille élémentaire en fond de fissure. Cette charge $P_{0}$, dépendant du maillage, n'a pas en elle-même une grande signification. On peut remarquer que les zones plastiques sont un peu plus grandes à l'extérieur qu'à l'intérieur.
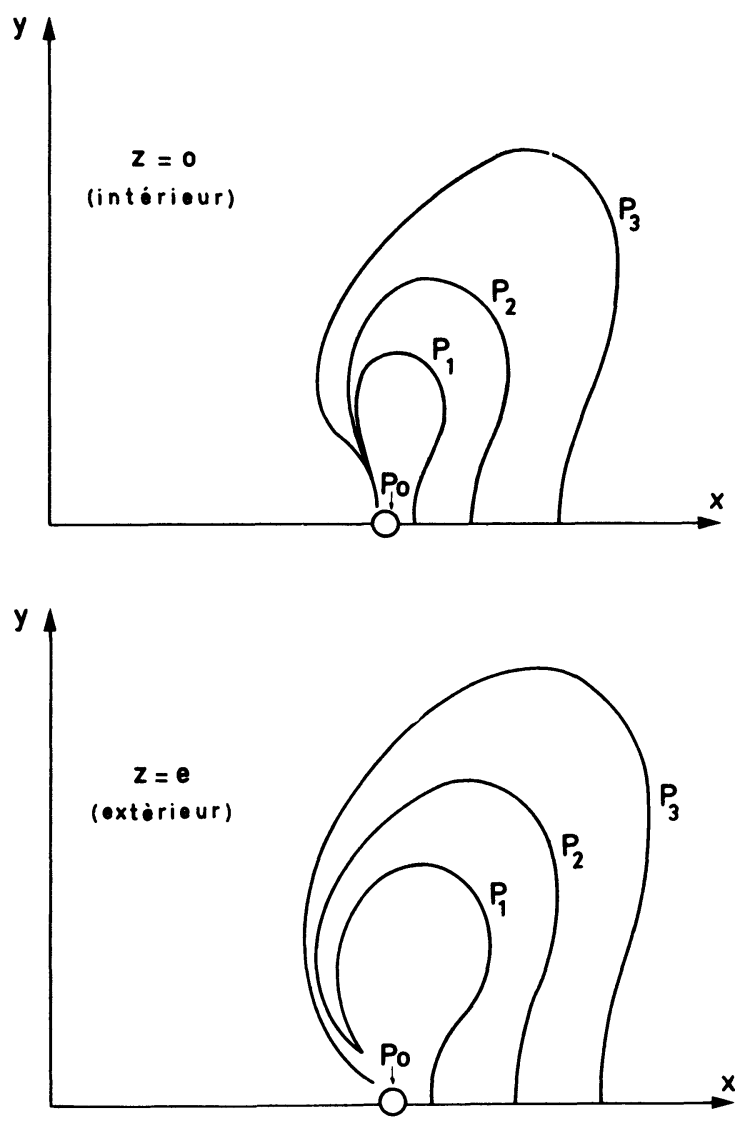

FIG. 5.

Dans l'éprouvette en flexion pure, il y a deux zones plastiques l'une en fond de fissure et l'autre dans la partie comprimée. Jusqu'à la valeur du couple $M_{0}$, à laquelle débute la plastification d'une éprouvette géométriquement identique mais non fissurée, les deux zones plastiques sont séparées. Jusqu'à cette valeur $M_{0}$, le champ de contrainte est voisin de la solution élastique et la variation du centre de rotation $\Omega$ est faible. Mais ce dernier varie beaucoup plus avec la charge lorsque $n$ est très grand (module d'écrouissage faible) ou encore lorsque le couple est plus élevé. Ce fait rend un peu délicate la corrélation entre le C. O. D. conventionnel en fond de fissure et le C. O. D. mesuré extérieurement plus loin de ce fond de fissure.

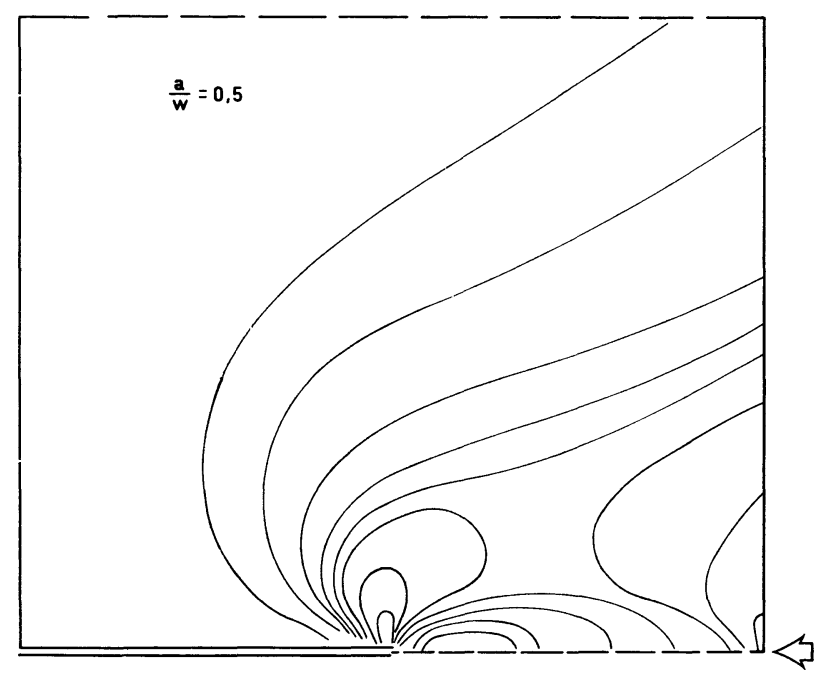

Fig. 6. - Zone plastique en flexion 3 points.

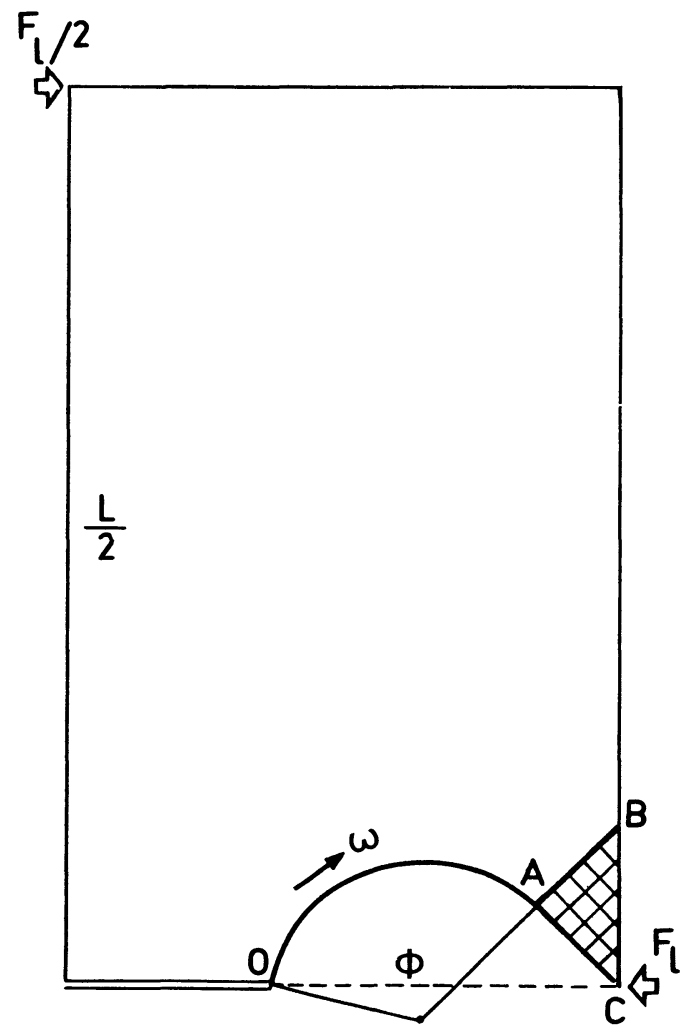

FIG. 7. 
Dans l'éprouvette en flexion à trois appuis, figure 6, les zones plastiques ont des allures différentes, avec un rétrécissement sous l'appui central. Les zones plastiques, de part et d'autre de l'appui central rappellent un peu les zones triangulaires de la solution cinématique en plasticité plane (matériau rigide-plastique) figure 7 .

Il est intéressant de rappeler pour comparaison cette solution cinématique qui fournit une borne supérieure de la charge limite $F_{1} \simeq \pi(1) 4 e / L$, avec $L$ longueur entre les deux appuis extérieurs, $2 e$ épaisseur et $\pi(\omega)$ la puissance dissipée pour la vitesse de rotation angulaire $\omega$. Cette puissance, qui dépend de l'angle $\varphi$ de l'arc de glissement, a été minimisée par rapport à $\varphi$ et c'est la valeur optimum réalisée pour $\varphi=1,36$ radian et pour $\omega=1$ qui est désignée par $\pi(1)$

$$
\pi(\omega)=2 k \omega\left[\frac{1}{2} \overline{A C}^{2}+R x(O A+A C)\right]
$$

$k=\sigma_{\mathrm{R} / \sqrt{3}}, \sigma_{\mathrm{R}}:$ charge de rupture en traction. Le calcul de la force limite $F_{1}$ s'appuyant sur des données réelles donne une valeur comparable à celle obtenue par le programme élasto-plastique tridimensionnel, bien que la nature du comportement ne soit pas la même dans les deux cas, seule la valeur de $\sigma_{\mathrm{R}}$ étant commune. Cela montre combien peut être utile un calcul de charge limite dans notre problème.

L'application de la mécanique «linéaire» de la rupture à des éprouvettes normalisées suppose que leur épaisseur est suffisante pour que l'état de déformation plane soit respecté sur la plus grande tranche possible. Les calculs numériques de $K_{I}$, trouvés dans la littérature, sont réalisés par des programmes bidimensionnels. Il est important de savoir si un calcul tridimensionnel élastique ne donnerait pas des valeurs de $K_{I}$ fondamentalement différentes. L'analyse de nos résultats numériques montre que la contrainte normale en fond de fissure (contraintes moyennes dans une maille élémentaire) est stationnaire sur $80 \%$ environ de l'épaisseur, puis diminue vers les deux faces non chargées, de $15 \%$ à $20 \%$ par rapport à la valeur au centre $(z=0)$. La valeur moyenne $\left\langle K_{I}\right\rangle$, comparée à $K_{I}$ obtenue au centre, est plus faible de 2 à $3 \%$ suivant le rapport a/e. Une autre valeur moyenne peut être définie, par la formule:

$$
\frac{1-v^{2}}{E} \bar{K}_{I}^{2}=\frac{\mathrm{d} W_{3}}{\mathrm{~d} l}
$$

où $W_{3}$ est l'énergie élastique obtenue par le calcul tridimensionnel. L'écart entre $\bar{K}_{I}$ et $K_{I}$ est supérieur à $5 \%$ pour l'éprouvette en flexion à trois appuis. Cet écart plus grand, peut s'expliquer par la présence des trois appuis. Il y a variation à travers l'épaisseur de toutes les singularités présentes : le fond de fissure et les appuis rectilignes.

\section{Bibliographie}

[1] Rice, J. R., J. Appl. Mech. 35 (1968) 379.

[2] Rice, J. R., Fracture 2 (Academic Press New-York, Londres) 1968, p. 191

[3] BuI, H. D., C. R. Hebd. Séan. Acad. Sci. 276A (1973) 1425.

[4] Griffith, A. A., Philo. Trans. Roy. Soc. (London) A 221 (1920) 163.

[5] Goodier, J. N., Fracture 2 (Academic Press New York et Londres), 1968, p. 2.
[6] Anderson, H. et Bergkivist, H., J. Mech. Phys. Solids 18 (1970) 1.

[7] BuI, H. D., Int. J. Solids Struct. 4 (1968) 1025.

[8] Mandel, J., Mécanique des Milieux Continus (GauthierVillars Paris) 1966, p. 706.

[9] Bui, H. D. et Nguyen, Q. S., Colloque Rupture (Grenoble) 1972.

[10] Bui, H. D. et NGuyen, Q. S., Engng. Fract. Mech. 5 (1973) 509.

[11] Ayres, D. J., Engng. Fract. Mech. 2 (1970) 87. 